\title{
Perspective projections in the space-frequency plane and fractional Fourier transforms
}

\author{
I. Şamil Yetik, Haldun M. Ozaktas, Billur Barshan, and Levent Onural \\ Department of Electrical Engineering, Bilkent University, TR-06533 Bilkent, Ankara, Turkey
}

Received March 16, 2000; revised manuscript received July 6, 2000; accepted July 10, 2000

\begin{abstract}
Perspective projections in the space-frequency plane are analyzed, and it is shown that under certain conditions they can be approximately modeled in terms of the fractional Fourier transform. The region of validity of the approximation is examined. Numerical examples are presented. (C) 2000 Optical Society of America [S0740-3232(00)00612-8]
\end{abstract}

OCIS codes: $100.0100,150.0150$.

\section{INTRODUCTION}

Perspective projections are used in many applications in image and video processing, especially when one is confronted with natural or artificial scenes with depth (for instance, in robot vision applications). Perspective projection can be considered as a geometric or pointwise transformation in the sense that each point of the object is mapped to another point in the perspective projection. $^{1-3}$ In this paper we examine the perspective projection in the space-frequency plane and show that its effect on the object can be modeled in terms of the fractional Fourier transform.

A widely employed space-frequency representation is the Wigner distribution, defined as

$$
\begin{aligned}
W_{f}\left(x, \sigma_{x}\right)= & \int f\left(x+x^{\prime} / 2\right) f^{*}\left(x-x^{\prime} / 2\right) \\
& \times \exp \left(-i 2 \pi \sigma_{x} x^{\prime}\right) \mathrm{d} x^{\prime} .
\end{aligned}
$$

The Wigner distribution provides information regarding the distribution of the signal energy over space and frequency. Especially important among its properties are the following relations:

$$
\begin{gathered}
\int W_{f}\left(x, \sigma_{x}\right) \mathrm{d} \sigma_{x}=|f(x)|^{2}, \\
\int W_{f}\left(x, \sigma_{x}\right) \mathrm{d} x=\left|F\left(\sigma_{x}\right)\right|^{2}, \\
\iint W_{f}\left(x, \sigma_{x}\right) \mathrm{d} x \mathrm{~d} \sigma_{x}=\|f\|^{2}=\operatorname{En}[f]=\text { signal energy. }
\end{gathered}
$$

The Wigner distribution of an exponential function $\exp (i 2 \pi \xi x)$ is a line delta lying parallel to the space axis:

$$
W_{f}\left(x, \sigma_{x}\right)=\delta\left(\sigma_{x}-\xi\right),
$$

and the Wigner distribution of a chirp function $\exp \left[i \pi\left(\chi x^{2}+2 \xi x+\zeta\right)\right]$ is an oblique line delta:

$$
W_{f}\left(x, \sigma_{x}\right)=\delta\left(\sigma_{x}-\chi x-\xi\right) .
$$

Further discussion regarding the properties of the Wigner distribution may be found in Ref. 4 .
The fractional Fourier transform is a generalization of the ordinary Fourier transform with a fractional order parameter $a$. It has found many applications in optics and signal processing. ${ }^{5-30}$ We refer the reader to Ref. 5 for a comprehensive treatment and further references and to Ref. 7 for a more concise introduction. Here we briefly mention a few important properties. The $a$ th-order fractional Fourier transform $f_{a}(u)$ is a unitary transform defined as

$$
\begin{aligned}
f_{a}(x)= & \int K_{a}\left(x, x^{\prime}\right) f\left(x^{\prime}\right) \mathrm{d} x^{\prime}, \\
K_{a}\left(x, x^{\prime}\right)= & A_{a} \exp \left\{i \pi \left[\cot (a \pi / 2) x^{2}\right.\right. \\
& \left.\left.-2 \csc (a \pi / 2) x x^{\prime}+\cot (a \pi / 2) x^{\prime 2}\right]\right\},
\end{aligned}
$$

where $A_{a}$ is a constant that depends on $a$. The zerothorder fractional Fourier transform corresponds to the identity operation, and the first-order fractional Fourier transform corresponds to the ordinary Fourier transform. Furthermore, the fractional Fourier transform is index additive; that is, the $a_{1}$ th-order fractional Fourier transform of the $a_{2}$ th-order fractional Fourier transform is equal to the $\left(a_{1}+a_{2}\right)$ th-order fractional Fourier transform. The $a$ th-order fractional Fourier transform corresponds to a clockwise rotation of the Wigner distribution by an angle $\alpha=a \pi / 2$ in the space-frequency plane:

$$
W_{f_{a}}\left(x, \sigma_{x}\right)=W_{f}\left(x \cos \alpha-\sigma_{x} \sin \alpha, x \sin \alpha+\sigma_{x} \cos \alpha\right) .
$$

The fractional Fourier transform has a fast implementation with complexity $O(N \log N) .^{5,7}$

To understand why the fractional Fourier transform is expected to play a role in perspective projections, let us consider the perspective projection of an image exhibiting periodic features, such as a railroad track. More distant parts of the image will appear smaller in the projection than closer parts will. Thus a periodic or harmonic feature of a certain frequency will be mapped such that it exhibits a monotonically increasing frequency. Under certain conditions this increase can be assumed linear so that the harmonic function is mapped to a chirp function. Since fractional Fourier transforms are known to map 
harmonic functions to chirp functions, we expect that perspective projections can be modeled in terms of fractional Fourier transforms. The purpose of this paper is to formulate this relationship.

In Section 2 we present the perspective model that we use and examine the effect of the perspective projection on the Wigner distribution. In Section 3 we discuss the relationship between the fractional Fourier transform and perspective projections based on their effects on the Wigner distribution. We also discuss how perspective projections can be modeled as shifted and fractional Fourier transformations. Section 4 is devoted to an analysis of the errors and the region of validity of the approximations.

\section{PERSPECTIVE PROJECTION}

The perspective model that we use is shown in Fig. 1. Initially, we consider perspective projections for onedimensional (1D) signals, since this approach significantly simplifies the presentation. The horizontal axis, labeled $x$, represents the original object space. The vertical axis, labeled $x_{p}$, represents the perspective projection space. The point A with coordinates $\left(-x_{0}, x_{p_{0}}\right)$ is the center of projection. We denote the original signal (object) by $f(x)$ and its perspective projection by $g\left(x_{p}\right)$. We assume that most of the energy of $f(x)$ is confined to the interval $[\bar{x}-\Delta x / 2, \bar{x}+\Delta x / 2]$. In the frequency domain we assume that most of the energy of $F\left(\sigma_{x}\right)$, the Fourier transform of $f(x)$, is confined to the interval [ $\bar{\sigma}_{x}$ $\left.-\Delta \sigma_{x} / 2, \bar{\sigma}_{x}+\Delta \sigma_{x} / 2\right]$. The value of $f(x)$ at each $x$ is mapped to the coordinate $x_{p}$, which is the projection of the point $x$ :

$$
\begin{gathered}
x_{p}=\frac{x x_{p_{0}}}{x+x_{0}}, \\
x=\frac{x_{0} x_{p}}{x_{p_{0}}-x_{p}},
\end{gathered}
$$

which can be derived by simple geometry. Thus the projection $g\left(x_{p}\right)$ is expressed as follows:

$$
g\left(x_{p}\right)=f\left(\frac{x_{0} x_{p}}{x_{p_{0}}-x_{p}}\right) .
$$

The interval to which most of the energy of $g\left(x_{p}\right)$ is approximately confined can be determined from Eq. (9).

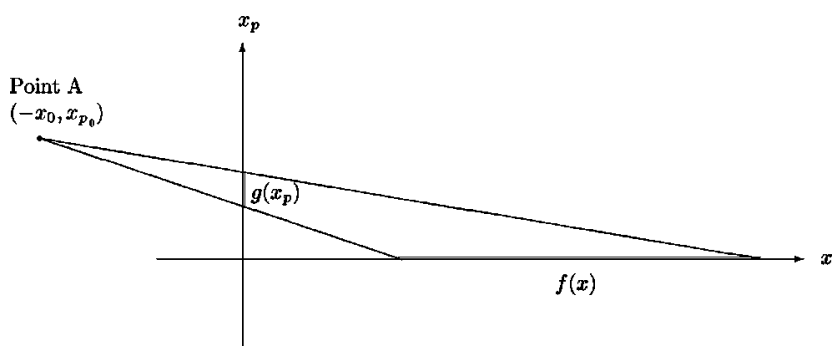

Fig. 1. Perspective model: $f(x)$ represents the object distribution on the $x$ axis; $g\left(x_{p}\right)$ represents its perspective projection onto the $x_{p}$ axis. The point $A$ with coordinates $\left(-x_{0}, x_{p_{0}}\right)$ is the center of projection.
To see the effect of perspective projections in the spacefrequency plane, we decompose $f(x)$ into harmonics as follows:

$$
f(x)=\int F\left(\sigma_{x}\right) \exp \left(i 2 \pi x \sigma_{x}\right) \mathrm{d} \sigma_{x},
$$

where $F\left(\sigma_{x}\right)$ is the Fourier transform of $f(x)$. Using Eq. (11) and the rules of linearity, we obtain the following expression for $g\left(x_{p}\right)$ :

$$
g\left(x_{p}\right)=\int F\left(\sigma_{x}\right) h\left(x_{p}, \sigma_{x}\right) \mathrm{d} \sigma_{x}
$$

where

$$
h\left(x_{p}, \sigma_{x}\right)=\exp \left[i 2 \pi \sigma_{x}\left(\frac{x_{0} x_{p}}{x_{p_{0}}-x_{p}}\right)\right] \mathrm{d} \sigma_{x} .
$$

We will initially concentrate on a single exponential with frequency $\bar{\sigma}_{x}$ and study the effect of perspective projection in the space-frequency plane. Then we will construct $g\left(x_{p}\right)$ by first decomposing $f(x)$ in terms of exponentials and using Eq. (13).

The Wigner distribution of $h\left(x_{p}, \bar{\sigma}_{x}\right)$ cannot be explicitly obtained. Therefore, to continue our analytical development, we expand the phase of $h\left(x_{p}, \bar{\sigma}_{x}\right)$ into a Taylor series. We will expand the phase of $h\left(x_{p}, \bar{\sigma}_{x}\right)$ around the point to which $\bar{x}$ is mapped:

$$
\frac{\bar{x}}{\bar{x}+x_{0}} x_{p_{0}},
$$

which we express as $\kappa x_{p_{0}}$, where $\kappa=\bar{x} /\left(\bar{x}+x_{0}\right)$. Expanding the phase of $h\left(x_{p}, \bar{\sigma}_{x}\right)$ around $\kappa x_{p_{0}}$, we obtain the following equation after performing some algebra:

$$
\begin{aligned}
h\left(x_{p}, \widetilde{\sigma}_{x}\right)= & \exp \left\{i 2 \pi \sigma _ { x } x _ { 0 } \left[\frac{x_{p}{ }^{2}}{(1-\kappa)^{3} x_{p_{0}}{ }^{2}}+\frac{x_{p}(1-3 \kappa)}{(1-\kappa)^{3} x_{p_{0}}}\right.\right. \\
& \left.\left.+\frac{\kappa^{3}}{(1-\kappa)^{3}}+\ldots\right]\right\} .
\end{aligned}
$$

Ignoring terms higher than the second order, we can see that the projection of a harmonic is a chirp function. The validity of this approximation requires that the thirdorder term be much smaller than the second-order term:

$$
|\kappa+2| \ll\left|2 x_{p_{0}}(\kappa-1)\right| \text {. }
$$

This approximation is more accurate for larger values of $x_{p_{0}}$. This is expected, since larger $x_{p_{0}}$ values correspond to perspective projections that are not as deep. The Wigner distribution of the chirp given in Eq. (16) is a line delta given by

$$
\delta\left[\sigma_{x}+\frac{2 \bar{\sigma}_{x}}{(1-\kappa)^{3} x_{p_{0}}{ }^{2}} x_{p}+\frac{\bar{\sigma}_{x}(1-3 \kappa)}{(1-\kappa)^{3} x_{p_{0}}}\right]
$$

and is shown in Fig. 2(b) below.

Having obtained an approximate analytical form for the perspective projection of a harmonic as well as an approximate expression for the perspective projection's Wigner distribution, we now move on to our discussion of 
perspective projections in the space-frequency plane and of their relationship to the fractional Fourier transform.

\section{RELATIONSHIP BETWEEN PERSPECTIVE PROJECTIONS AND FRACTIONAL FOURIER TRANSFORMS}

In Section 2 we obtained an approximate expression for the Wigner distribution of the perspective projection of a single exponential. The Wigner distribution of a typical exponential and the Wigner distribution of the approximate perspective projection of the exponential are shown in Fig. 2. The angle that the line delta makes with the $x$ axis is $\arctan \left\{2 \bar{\sigma}_{x} /\left[(1-\kappa)^{3} x_{p_{0}}{ }^{2}\right]\right\}$ which depends on $\bar{\sigma}_{x}$. The fact that the oblique line delta is a rotated version of the horizontal line delta suggests a role for the fractional Fourier transform, since this operation corresponds to rotation in the space-frequency plane.

We will now show how the perspective projection of a signal can be approximately expressed in terms of the fractional Fourier transform. We claim that the perspective projection of a signal can be obtained from, or modeled by, the following steps:

1. Shift the signal by $\bar{x}$ in the negative $x$ direction and by $\bar{\sigma}_{x}$ in the negative $\sigma_{x}$ direction. This translates the Wigner distribution of the signal to the origin of the space-frequency plane.

2. Take the fractional Fourier transform with the order $a=(-2 / \pi) \arctan \left\{\left[2 \bar{\sigma}_{x}\left(\bar{x}+x_{0}\right)^{3}\right] / x_{p_{0}}{ }^{2} x_{0}{ }^{2}\right\}$. This rotates the Wigner distribution by an angle $a \pi / 2$.

3. Shift the result by $\bar{x} x_{p_{0}} /\left(\bar{x}+x_{0}\right)$ in the positive $x$ direction and by $\left[\bar{\sigma}_{x}\left(\bar{x}+x_{0}\right)^{2}\right] / x_{0} x_{p_{0}}$ in the positive $\sigma_{x}$ direction.

These steps represent a decomposition of the overall effect of the perspective projection, from which we can see that to perform perspective projection is essentially to effect a rotation in the space-frequency plane. However, this rotation is enacted on the space-frequency content of the signal that is referred to the origin of the spacefrequency plane. The above steps are illustrated in Fig. 3.

Different frequency components of the signal require different fractional Fourier orders, because the order $a$ given in step 3 depends on $\bar{\sigma}_{x}$. However, as we will show, under certain conditions a satisfactory approximation can be obtained by use of a uniform order corresponding to the central frequency of the signal.

We now demonstrate our claim that perspective projection can be decomposed into the three steps given above. We start by decomposing $f(x)$ into harmonics:

$$
f(x)=\int F\left(\sigma_{x}\right) \exp \left(2 i \pi x \sigma_{x}\right) \mathrm{d} \sigma_{x} .
$$

We will concentrate on a single-harmonic component, $\exp \left(i 2 \pi x \sigma_{x}\right)$, and the result for general $f(x)$ will follow by linearity. Applying step 1 to a single harmonic, we obtain

$$
\exp \left(i 2 \pi \bar{x} \sigma_{x}\right)
$$

Now we apply steps 2 and 3 to this result to obtain

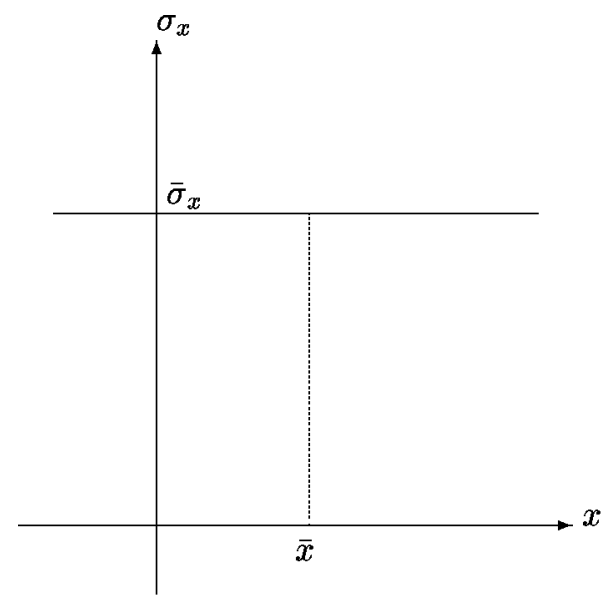

(a)

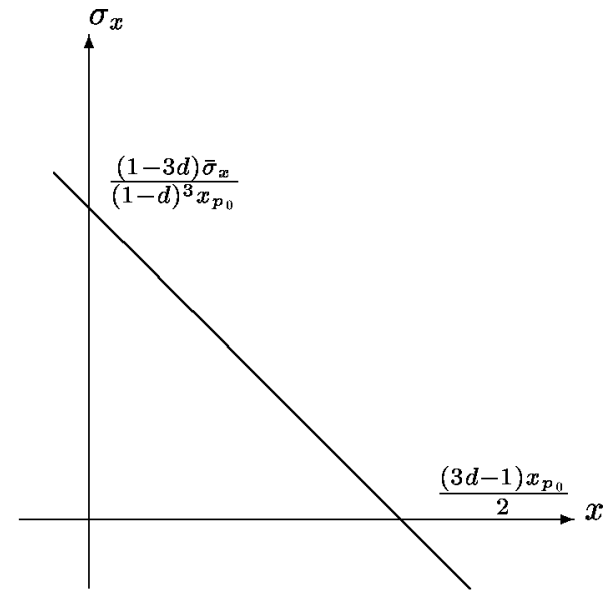

(b)

Fig. 2. (a) Wigner distribution of the original exponential. (b) Wigner distribution of the approximate perspective projection: a chirp.

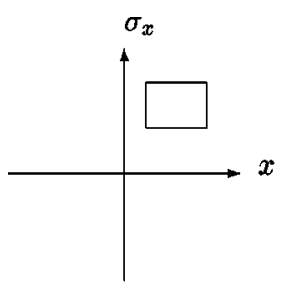

(a)

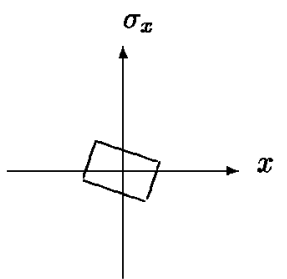

(c)

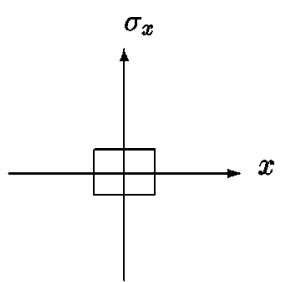

(b)

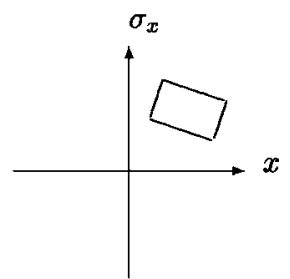

(d)
Fig. 3. Illustration of the decomposition of the approximation into elementary operations in the space-frequency plane: (a) Original signal, (b) after step 1 (space and frequency shift), (c) after step 2 (fractional Fourier transform), (d) after step 3 (space and frequency shift): approximate perspective projection. 


$$
\begin{gathered}
\left(1+i \frac{2 \sigma_{x} \bar{x}+x_{0}}{x_{p_{0}}{ }^{2} x_{0}{ }^{3}}\right)^{1 / 2} \exp \left(i 2 \pi \bar{x} \sigma_{x}\right) \\
\quad \times \exp \left[\left(i 2 \pi x^{2} \sigma_{x} \frac{\bar{x}+x_{0}}{x_{p_{0}}{ }^{2} x_{0}{ }^{3}}\right)\right] .
\end{gathered}
$$

Last, we apply step 4 and obtain our final result:

$$
\begin{aligned}
{[1+} & \left.i \frac{2 \sigma_{x}\left(\bar{x}+x_{0}\right)^{3}}{x_{p_{0}}{ }^{2} x_{0}{ }^{3}}\right]^{1 / 2} \exp \left(i 2 \pi \bar{x} \sigma_{x}\right) \\
& \times \exp \left[i 2 \pi \sigma_{x}\left(x-\frac{\bar{x} x_{p_{0}}}{\bar{x}+x_{0}}\right)^{2}\left(\frac{\bar{x}+x_{0}}{x_{p_{0}}{ }^{2} x_{0}{ }^{3}}\right)\right] \\
& \times \exp \left[i 4 \pi \sigma_{x}\left(\frac{\bar{x}+x_{0}{ }^{2}}{x_{0}{ }^{2} x_{p_{0}}}\right)\right] .
\end{aligned}
$$

Multiplying this by $F\left(\sigma_{x}\right)$ and integrating over $\sigma_{x}$ yields the desired approximate expression for the perspective projection of $f(x)$, which is the mathematical expression of the four steps outlined above.

To see that this expression is indeed an approximation of the perspective projection, we again concentrate on a single-harmonic component whose exact perspective projection is

$$
\exp \left(2 i \pi \sigma_{x} \frac{x_{0} x_{p}}{x_{p_{0}}-x_{p}}\right)
$$

Using the Taylor-series expansion, we obtain

$$
\begin{aligned}
& \exp \left\{i 2 \pi \sigma _ { x } x _ { 0 } \left[\frac{x_{p}{ }^{2}[)^{3} x_{p_{0}}{ }^{2}}{\left(1-\frac{x_{p}(1-3 \kappa)}{(1-\kappa)^{3} x_{p_{0}}}\right.}\right.\right. \\
& \left.\left.+\frac{\kappa^{3}}{(1-\kappa)^{3}}\right]\right\},
\end{aligned}
$$

which differs from expression (22) only by a constant factor. As far as a single-harmonic component is concerned, the only approximation that is involved is the binomial expansion in the exponent. When the harmonic components are superposed to yield the original function $f(x)$, we make the additional approximation of using the order corresponding to the center frequency for all the harmonic components. Thus our three-step procedure will deviate from the exact perspective projection more and more as the bandwidth of $f(x)$ is increased. The limitations associated with this approximation are discussed in Section 4.

Figure 4 shows the exact perspective projection of the function

$$
\begin{aligned}
\cos (4 \pi x) \operatorname{rect}\left(\frac{x-4}{6}\right)= & {\left[\frac{\exp (i 4 \pi x)+\exp (-i 4 \pi x)}{2}\right] } \\
& \times \operatorname{rect}\left(\frac{x-4}{6}\right)
\end{aligned}
$$

superimposed upon the approximation given by expression (24). We chose $x_{0}=-3, x_{p_{0}}=6$ as the center of projection. As a second example, we consider the narrow-band signal shown in Fig. 5. Here, too, the exact perspective projection and the fractional Fourier approximation are superimposed [see Fig. 5(b)]. We observe that the approximation is quite satisfactory except for very near the edges, which should be avoided.

Generalization of the proposed method to two dimensions is possible if similar steps are followed. In our twodimensional (2D) perspective model we use a 2D image with midpoints $\bar{x}, \bar{y}$; center frequencies $\bar{\sigma}_{x}, \bar{\sigma}_{y}$; and spatial widths $\Delta x, \Delta y$. Our center of projection is located at $\left(x_{0}, x_{p_{0}}, 0\right)$. The model described is shown in Fig. 6 . With this model, using simple geometry, we can obtain the following mappings and reverse mappings for each $x_{p}$ and $y_{p}$ :

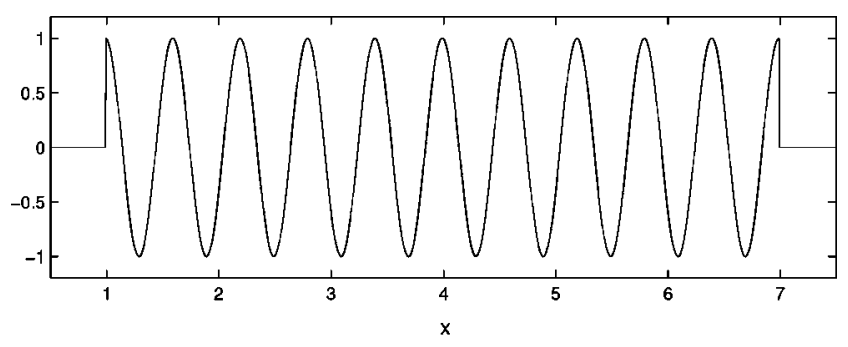

(a)

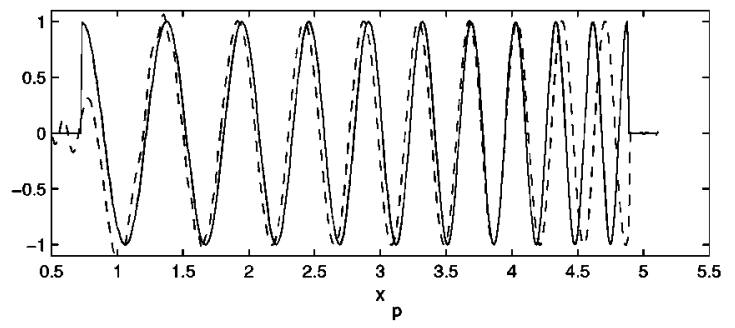

(b)

Fig. 4. (a) Original signal. (b) Exact perspective projection (solid curve) superimposed upon the fractional Fourier approximation (dashed curve).

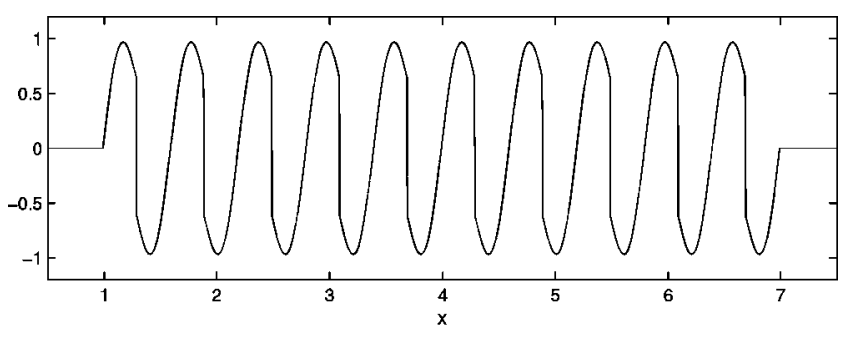

(a)

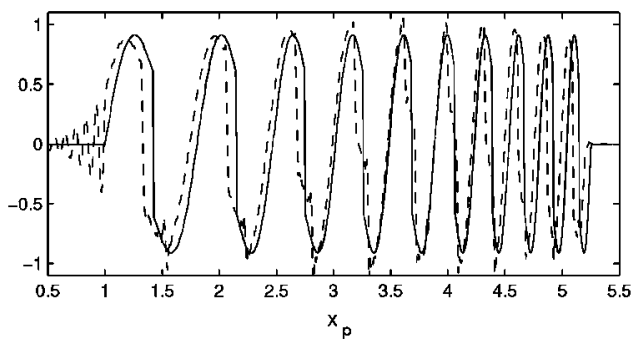

(b)

Fig. 5. (a) Original signal. (b) Exact perspective projection (solid curve) superimposed upon the fractional Fourier approximation (dashed curve). 


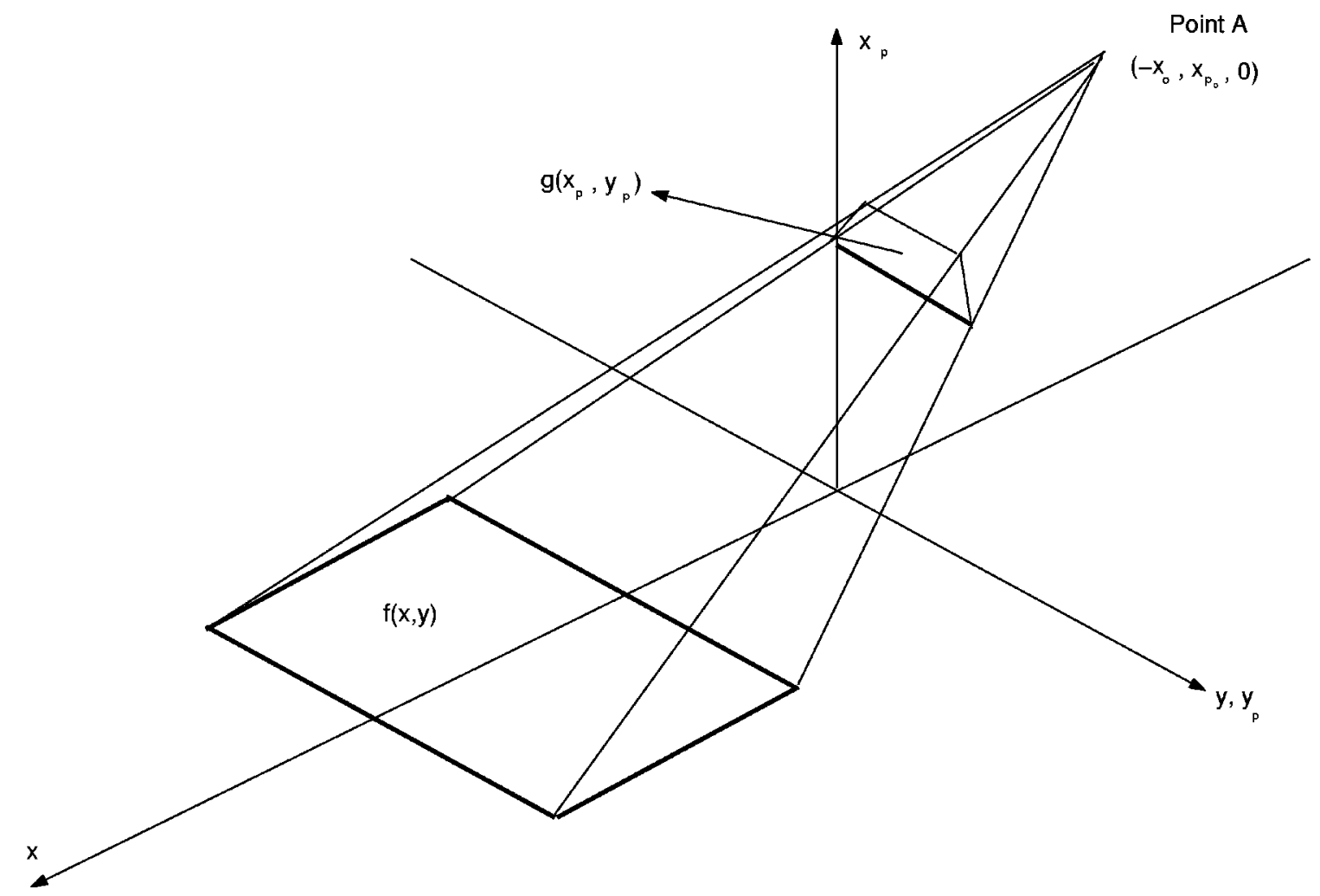

Fig. 6. Perspective model: $f(x, y)$ represents the object distribution on the $x-y$ plane; $g\left(x_{p}, y_{p}\right)$ represents the object distribution's perspective projection onto the $x_{p}-y_{p}$ plane. The point $A$ with coordinates $\left(-x_{0}, x_{p_{0}}, 0\right)$ is the center of projection.

$$
\begin{aligned}
& x_{p}=\frac{x x_{p_{0}}}{x+x_{0}}, \\
& x=\frac{x_{0} x_{p}}{x_{p_{0}}-x_{p}}, \\
& y_{p}=\frac{x \Delta y+2 x_{0} y}{2\left(x+x_{0}\right)}, \\
& y=\frac{y_{p} x_{p_{0}}}{x_{p_{0}}-x_{p}}-\frac{x_{p} \Delta y}{2\left(x_{0}-x_{p}\right)} .
\end{aligned}
$$

As in the $1 \mathrm{D}$ case, we first decompose $f(x, y)$ into harmonics:

$f(x, y)=\int F\left(\sigma_{x}, \sigma_{y}\right) \exp \left(i 2 \pi \sigma_{x} x\right) \exp \left(i 2 \pi \sigma_{y} y\right) \mathrm{d} \sigma_{x} \mathrm{~d} \sigma_{y}$.

We proceed by writing an expression for the perspective projection of a $2 \mathrm{D}$ harmonic, $\exp \left(i 2 \pi \bar{\sigma}_{x} x\right) \exp \left(i 2 \pi \bar{\sigma}_{y} y\right)$ :

$$
\begin{aligned}
\exp \{ & i 2 \pi \bar{\sigma}_{x} x_{0}\left[\frac{x_{p}{ }^{2}}{(1-\kappa)^{3} x_{p_{0}}{ }^{2}}+\frac{x_{p}(1-3 \kappa)}{(1-\kappa)^{3} x_{p_{0}}}\right. \\
& \left.\left.+\frac{\kappa^{3}}{(1-\kappa)^{3}}\right]\right\}, \\
& \times \exp \left\{-i \pi \bar{\sigma}_{y} \Delta y\left[\frac{x_{p}{ }^{2}}{\left(1-\kappa^{\prime}\right)^{3} x_{0}{ }^{2}}+\frac{x_{p}\left(1-3 \kappa^{\prime}\right)}{\left(1-\kappa^{\prime}\right)^{3} x_{0}}\right.\right. \\
& \left.\left.+\frac{\kappa^{\prime 3}}{\left(1-\kappa^{\prime}\right)^{3}}\right]\right\}, \\
& \times \exp \left\{i 2 \pi \overline { \sigma } _ { y } y _ { p } \left[\frac{x_{p}{ }^{2}}{x_{p_{0}}{ }^{2}(1-\kappa)^{3}}+\frac{x_{p}(1-3 \kappa)}{x_{p}(1-\kappa)^{3}}\right.\right. \\
& \left.\left.+\frac{3 \kappa^{2}-3 \kappa+1}{(\kappa-1)^{3}}\right]\right\},
\end{aligned}
$$

where again the binomial approximation has been employed and $\kappa=\bar{x} /\left(\bar{x}+x_{0}\right)$ and $\kappa^{\prime}=\bar{x} /(\bar{x}+\Delta y)$. Close examination of expression (31) reveals that we have the product of a $1 \mathrm{D}$ chirp in the $x_{p}$ direction and a scaled harmonic in the $y_{p}$ direction whose scaling factor depends on $x_{p}$. We are going to approximate the perspective projection by using $1 \mathrm{D}$ shifts and 1D fractional Fourier transforms followed by scaling. We claim that the $2 \mathrm{D}$ perspective projection of a signal can be obtained from, or modeled by, the following steps: 
1. Shift the signal by $\bar{x}$ in the negative $x$ direction and by $\bar{\sigma}_{x}$ in the negative $\sigma_{x}$ direction. This translates the Wigner distribution of the signal to the origin of the space-frequency plane.

2. Take the 1D fractional Fourier transforms in the variable $x$ with order

$$
a=\frac{-2}{\pi} \arctan \left[\frac{2 \bar{\sigma}_{x}\left(\bar{x}+x_{0}\right)^{3}}{x_{p_{0}}{ }^{2} x_{0}{ }^{2}}-\frac{(\bar{x}+\Delta y)^{3} \bar{\sigma}_{y}}{\Delta y^{2} x_{0}{ }^{2}}\right],
$$

treating $y$ as a parameter. This rotates the Wigner distributions by an angle $a \pi / 2$.

3. Shift the result by $\bar{x} x_{p_{0}} /\left(\bar{x}+x_{0}\right)$ in the positive $x$ direction and by

$$
\left[\frac{\bar{\sigma}_{x}\left(\bar{x}+x_{0}\right)^{3}}{x_{p_{0}} x_{0}}-\frac{(\bar{x}+\Delta y)^{3} \bar{\sigma}_{y}}{\Delta y^{2} x_{0}{ }^{2}}\right]
$$

in the positive $\sigma_{x}$ direction.

4. Scale each horizontal line of the perspective projection by

$$
\left\{\bar{\sigma}_{y}\left[\frac{x_{p}{ }^{2}}{x_{p_{0}}{ }^{2}(1-\kappa)^{3}}+\frac{x_{p}(1-3 \kappa)}{x_{p}(1-\kappa)^{3}}+\frac{3 \kappa^{2}-3 \kappa+1}{(\kappa-1)^{3}}\right]\right\} .
$$

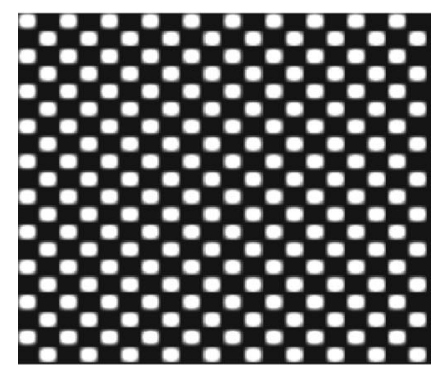

(a)

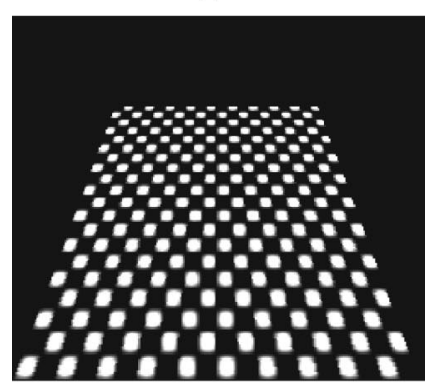

(b)

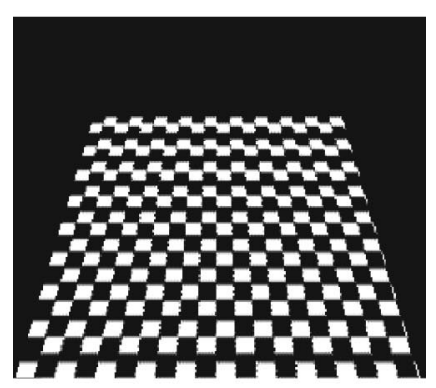

(c)

Fig. 7. (a) Original signal. (b) Exact perspective projection. (c) Fractional Fourier approximation.
The mathematical combination of the above steps yields the $2 \mathrm{D}$ perspective projection of a $2 \mathrm{D}$ harmonic, given by expression (31). Multiplying this by $F\left(\sigma_{x}, \sigma_{y}\right)$ and integrating over $\sigma_{x}$ and $\sigma_{y}$ yields the desired approximate expression for the perspective projection of $f(x, y)$, which is the mathematical expression of the four steps outlined above. An example is given in Fig. 7, where the fractional-Fourier-transform-based result shown in Fig. 7(c) can be seen to be a reasonable approximation of the actual perspective projection shown in Fig. 7(b).

\section{ERROR ANALYSIS}

In this section we examine the conditions under which the fractional Fourier transform approximation to the perspective projection is valid. We first examine the modifications that the Wigner distribution undergoes corresponding to the approximation. Since we know that the approximation can be decomposed into the four steps given in Section 3, it is easy to find the resulting changes in the Wigner distribution. To estimate the error inherent in our approximation, we will think of the original Wigner distribution as consisting of horizontal strips of narrow frequency components. The major approximation that we make is to replace the fractional orders required by these different frequency components with a single order corresponding to the central frequency. To determine the error introduced by this approximation, we first determine how the highest- and the lowest-frequency strips would be mapped had their individual frequencies been used instead of the center frequency. Let us assume that most of the energy of the Wigner distribution of a signal is concentrated in a rectangular region in the space-frequency plane [Fig. 8(a)]. Figure 8(b) shows the Wigner distribution corresponding to the fractional Fourier approximation (solid lines). The dashed lines, in contrast, show the Wigner contour obtained by using the individual frequencies for the highest- and the lowestfrequency strips.

Our error criteria will be the deviations of the corners of the two superimposed Wigner contours shown in [Fig. 8(b)]. There will be one spatial deviation and one frequency deviation for each of the four corners of the contours. We will normalize the spatial deviation by $\Delta x$ and the frequency deviations by $\Delta \sigma_{x}$ and take the maximum of the resulting eight normalized deviations as our final error measure. Expressions for the eight normalized deviations are given below:

$$
\begin{aligned}
e_{\text {up-left,space }}= & \frac{\Delta \sigma_{x}}{\Delta x x_{0}} \sin \left(\frac{\alpha_{c}-\alpha_{u}}{2}\right)\left[\cos \left(\frac{\alpha_{c}+\alpha_{u}}{2}\right)\right. \\
& \left.+\frac{1}{x_{0}} \sin \left(\frac{\alpha_{c}+\alpha_{u}}{2}\right)\right], \\
e_{\text {up-right,space }}= & \frac{\Delta \sigma_{x}}{\Delta x x_{0}} \sin \left(\frac{\alpha_{c}-\alpha_{u}}{2}\right)\left[\cos \left(\frac{\alpha_{c}+\alpha_{u}}{2}\right)\right. \\
& \left.-\frac{1}{x_{0}} \sin \left(\frac{\alpha_{c}+\alpha_{u}}{2}\right)\right],
\end{aligned}
$$




$$
\begin{aligned}
e_{\text {low-left,space }}= & -\frac{\Delta \sigma_{x}}{\Delta x x_{0}} \sin \left(\frac{\alpha_{c}-\alpha_{d}}{2}\right)\left[\cos \left(\frac{\alpha_{c}-\alpha_{d}}{2}\right)\right. \\
& \left.+\frac{1}{x_{0}} \sin \left(\frac{\alpha_{c}+\alpha_{d}}{2}\right)\right], \\
e_{\text {low-right,space }}= & -\frac{\Delta \sigma_{x}}{\Delta x x_{0}} \sin \left(\frac{\alpha_{c}-\alpha_{d}}{2}\right)\left[\cos \left(\frac{\alpha_{c}+\alpha_{d}}{2}\right)\right. \\
& \left.-\frac{1}{x_{0}} \sin \left(\frac{\alpha_{c}+\alpha_{d}}{2}\right)\right],
\end{aligned}
$$

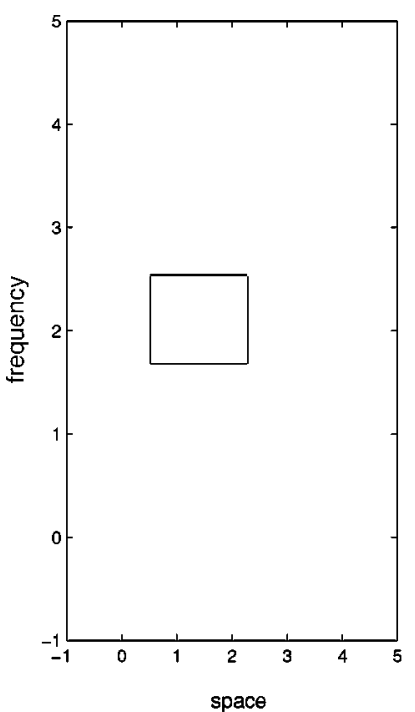

(a)

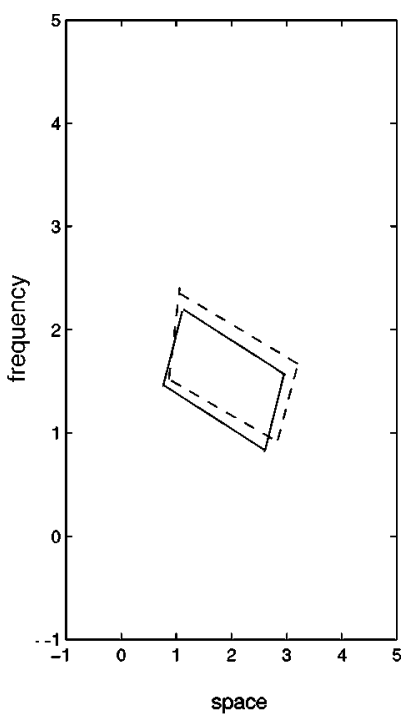

(b)

$$
\begin{aligned}
e_{\text {up-left,freq. }}=- & \frac{1}{x_{0}} \sin \left(\frac{\alpha_{c}-\alpha_{u}}{2}\right)\left[\sin \left(\frac{\alpha_{c}+\alpha_{u}}{2}\right)\right. \\
& \left.+\frac{\Delta x}{\Delta \sigma_{x} x_{0}} \cos \left(\frac{\alpha_{c}+\alpha_{u}}{2}\right)\right], \\
e_{\text {up-right,freq. }}= & -\frac{1}{x_{0}} \sin \left(\frac{\alpha_{c}-\alpha_{u}}{2}\right)\left[\sin \left(\frac{\alpha_{c}+\alpha_{u}}{2}\right)\right. \\
& \left.-\frac{\Delta x}{\Delta \sigma_{x} x_{0}} \cos \left(\frac{\alpha_{c}+\alpha_{u}}{2}\right)\right], \\
e_{\text {low-left,freq. }}= & \frac{1}{x_{0}} \sin \left(\frac{\alpha_{c}-\alpha_{d}}{2}\right)\left[\sin \left(\frac{\alpha_{c}+\alpha_{d}}{2}\right)\right. \\
& \left.+\frac{\Delta x}{\Delta \sigma_{x} x_{0}} \cos \left(\frac{\alpha_{c}+\alpha_{d}}{2}\right)\right] \\
& \left.-\frac{1}{\Delta \sigma_{x} x_{0}} \cos \left(\frac{\alpha_{c}+\alpha_{d}}{2}\right)\right], \\
e_{\text {low-right,freq. }}= & \left.\frac{\alpha_{c}-\alpha_{d}}{x_{0}}\right)\left[\sin \left(\frac{\alpha_{c}+\alpha_{u}}{2}\right)\right.
\end{aligned}
$$

where

$$
\alpha_{c}=\arctan \left[\frac{2 \frac{\bar{\sigma}_{x}}{x_{0}}\left(\frac{\bar{x}+x_{0}}{x_{0}}\right)^{3}}{\left(\frac{x_{p_{0}}}{x_{0}}\right)^{2}}\right] \text {, }
$$

parison of Wigner distributions underlying error analysis.

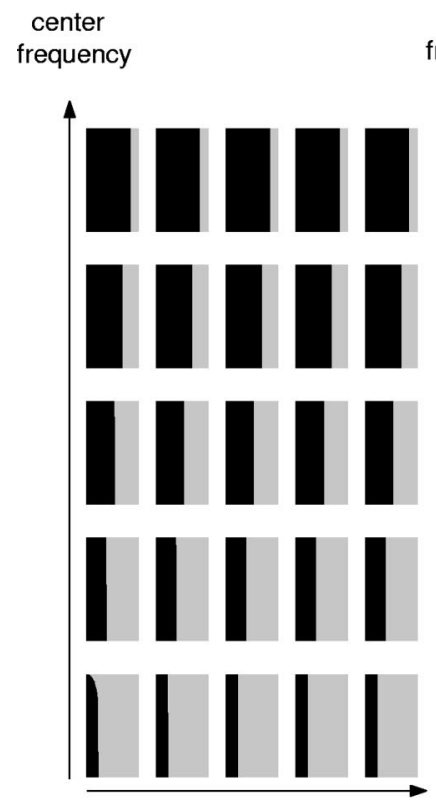

center point

(a) center
frequency
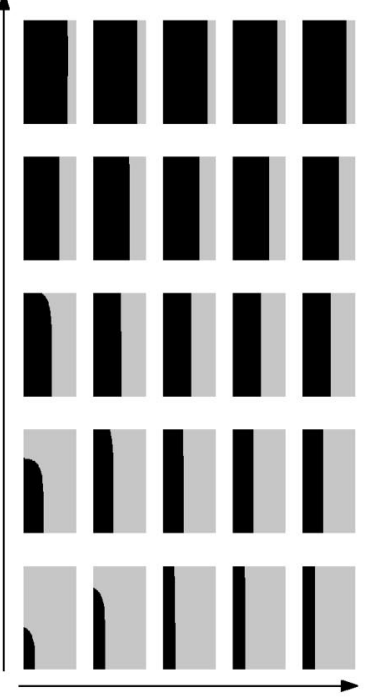

center point

(b) center frequency
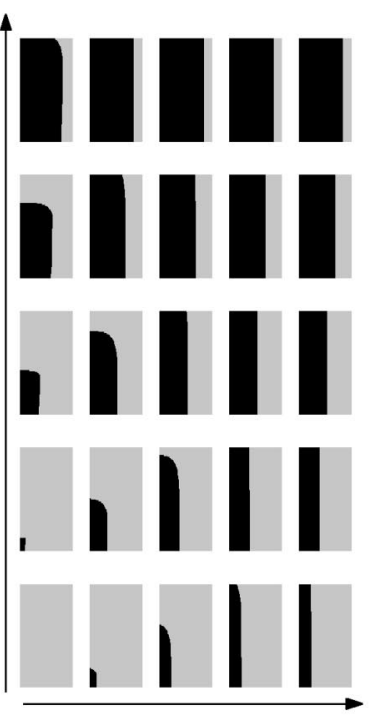

center point

(c)

Fig. 9. Dark shading: parameter combinations whose normalized error is less than $10 \%$. Light shading: region in which the error is large. See text for details. 


$$
\begin{aligned}
& \alpha_{u}=\arctan \left[\frac{2\left(\frac{\bar{\sigma}_{x}}{x_{0}}+\frac{\Delta \sigma_{x}}{2 x_{0}}\right)\left(\frac{\bar{x}+x_{0}}{x_{0}}\right)^{3}}{\left(\frac{x_{p_{0}}}{x_{0}}\right)^{2}}\right], \\
& \alpha_{d}=\arctan \left[\frac{2\left(\frac{\bar{\sigma}_{x}}{x_{0}}-\frac{\Delta \sigma_{x}}{2 x_{0}}\right)\left(\frac{\bar{x}+x_{0}}{x_{0}}\right)^{3}}{\left(\frac{x_{p_{0}}}{x_{0}}\right)^{2}}\right] .
\end{aligned}
$$

To reduce the number of parameters by one, we have expressed the above results so that all the free parameters appear divided by $x_{0}$.

It does not seem possible to analytically derive conclusions by use of these formulas, so we will resort to numerically obtained plots. The approximation will be assumed to be acceptable if the maximum normalized error is less than $10 \%$. The expressions given above yield the error as a function of six variables: $x_{0}, x_{p_{0}}, \bar{x}, \bar{\sigma}_{x}$, $\Delta x, \Delta \sigma_{x}$. However, normalizing all the variables by $x_{0}$, we can reduce the number of variables to five. In Fig. 9 we show as dark regions the region in which the maximum normalized error is less than $10 \%$, whereas the light regions indicate that in which the error is large. The horizontal axis in each of the 75 plots represents the value of $\Delta x / x_{0}$, and the vertical axis represents $\Delta \sigma_{x} / x_{0}$. Both these variables range from $10^{1 / 30}$ to $10^{100 / 30}$ in these $\log -\log$ plots.

Each member of the $5 \times 5$ matrices of plots corresponds to different values of $\bar{x} / x_{0}$ (horizontal), $\bar{\sigma}_{x} / x_{0}$ (vertical). The five separate values of $\bar{x} / x_{0}$ are $10^{-1 / 2}, 10^{0 / 2}$, $10^{1 / 2}, 10^{2 / 2}, 10^{3 / 2}$, and the five separate values of $\bar{\sigma}_{x} / x_{0}$ are $10^{-1 / 2}, 10^{0 / 2}, 10^{1 / 2}, 10^{2 / 2}, 10^{3 / 2}$. The three groups of 25 plots each correspond to different values of the center of projection: $\quad x_{p_{0}} / x_{0}=0.1$ [Fig. 9(a)], $x_{p_{0}} / x_{0}=1$ [Fig. 9(b)], $x_{p_{0}} / x_{0}=10$ [Fig. 9(c)].

This set of plots covering a broad range of the parameter values allows us to determine whether the approximation developed is acceptable for a certain range of parameters. Generally speaking, we have larger acceptable regions for larger values of $\bar{\sigma}_{x}$. Not surprisingly, the approximation is strained as $\Delta x$ and $\Delta \sigma_{x}$ increase, i.e., as the space-bandwidth product of the signal increases.

\section{CONCLUSION}

In this paper we examined perspective projections in the space-frequency plane and showed how to approximate the perspective projection in terms of the fractional Fourier transform. Our main motivation was to show that the fractional Fourier transform approximately captures the essence of the warping characteristic of perspective projections. We observed that perspective projection approximately maps harmonic components into chirps and that it can therefore be modeled in terms of the fractional Fourier transform. We saw that the substance of perspective projection is essentially to effect a rotation in the space-frequency plane. However, this rotation is enacted on the space-frequency content of the signal referred to the origin of the space-frequency plane. Elementary nu- merical examples for both one-dimensional signals and two-dimensional images were presented. The errors associated with the approximation and the region of validity with respect to the approximations involved were numerically discussed.

I. Ş. Yetik can be reached by e-mail at yetik@ee.bilkent.edu.tr.

\section{REFERENCES}

1. D. Vernon, Machine Vision: Automated Visual Inspection and Robot Vision (Prentice-Hall, New York, 1991)

2. A. Low, Introductory Computer Vision and Image Processing (McGraw-Hill, New York, 1991).

3. G. Woldberg, Digital Image Warping (IEEE Computer Society, Los Alamitos, Calif., 1992).

4. L. Cohen, Time-Frequency Analysis (Prentice-Hall PTR, Englewood Cliffs, N.J., 1995).

5. H. M. Ozaktas, Z. Zalevsky, and M. A. Kutay, The Fractional Fourier Transform with Applications in Optics and Signal Processing (Wiley, New York, 2000).

6. H. M. Ozaktas, B. Barshan, D. Mendlovic, and L. Onural, "Convolution, filtering, and multiplexing in fractional Fourier domains and their relation to chirp and wavelet transforms," J. Opt. Soc. Am. A 11, 547-559 (1994).

7. H. M. Ozaktas, M. A. Kutay, and D. Mendlovic, "Introduction to the fractional Fourier transform and its applications," Adv. Imaging Electron Phys. 106, 239-291 (1999).

8. L. B. Almeida, "The fractional Fourier transform and timefrequency representations," IEEE Trans. Signal Process. 42, 3084-3092 (1994).

9. S. Abe and J. T. Sheridan, "Comment on "The fractional Fourier transform in optical propagation problems,'” J. Mod. Opt. 42, 2373-2378 (1995).

10. O. Akay and G. F. Boudreaux-Bartels, "Unitary and Hermitian fractional operators and their relation to the fractional Fourier transform," IEEE Signal Process. Lett. 5, 312-314 (1998).

11. T. Alieva, "Fractional Fourier transform as a tool for investigation of fractal objects," J. Opt. Soc. Am. A 13, 11891192 (1996).

12. T. Alieva and F. Agullo-Lopez, "Diffraction analysis of random fractal fields,” J. Opt. Soc. Am. A 15, 669-674 (1998).

13. L. M. Bernardo and O. D. D. Soares, "Fractional Fourier transforms and imaging," J. Opt. Soc. Am. A 11, 2622-2626 (1994); Y. Bitran, Z. Zalevsky, D. Mendlovic, and R. G. Dorsch, "Fractional correlation operation: performance analysis," Appl. Opt. 35, 297-303 (1996).

14. W. X. Cong, N. X. Chen, and B. Y. Gu, "Beam shaping and its solution with the use of an optimization method," Appl. Opt. 37, 4500-4503 (1998).

15. R. G. Dorsch and A. W. Lohmann, "Fractional Fourier transform used for a lens design problem," Appl. Opt. 34, 4111-4112 (1995)

16. D. Dragoman and M. Dragoman, "Near and far field optical beam characterization using the fractional Fourier transform," Opt. Commun. 141, 5-9 (1997).

17. M. F. Erden, M. A. Kutay, and H. M. Ozaktas, "Repeated filtering in consecutive fractional Fourier domains and its application to signal restoration," IEEE Trans. Signal Process. 47, 1458-1462 (1999).

18. J. García, D. Mendlovic, Z. Zalevsky, and A. Lohmann, "Space-variant simultaneous detection of several objects by the use of multiple anamorphic fractional-Fouriertransform filters," Appl. Opt. 35, 3945-3952 (1996).

19. S. Granieri, O. Trabocchi, and E. E. Sicre, "Fractional Fourier transform applied to spatial filtering in the Fresnel domain," Opt. Commun. 119, 275-278 (1995).

20. C. J. Kuo and Y. Luo, "Generalized joint fractional Fourier transform correlators: a compact approach," Appl. Opt. 37, 8270-8276 (1998). 
21. M. A. Kutay and H. M. Ozaktas, "Optimal image restoration with the fractional Fourier transform," J. Opt. Soc. Am. A 15, 825-833 (1998).

22. M. A. Kutay, H. M. Ozaktas, O. Arikan, and L. Onural, "Optimal filtering in fractional Fourier domains," IEEE Trans. Signal Process. 45, 1129-1143 (1997).

23. A. W. Lohmann, D. Mendlovic, and Z. Zalevsky, "Fractional transformations in optics," in Progress in Optics, XXXVIII, E. Wolf, ed. (Elsevier, Amsterdam, 1998), Chap. 4, pp. 263342 .

24. S. Mann and S. Haykin, "The chirplet transform: physical considerations," IEEE Trans. Signal Process. 43, 27452761 (1995)

25. D. Mendlovic, Z. Zalevsky, A. W. Lohmann, and R. G. Dorsch, "Signal spatial-filtering using the localized frac- tional Fourier transform," Opt. Commun. 126, 14-18 (1996).

26. V. Namias, "The fractional order Fourier transform and its application to quantum mechanics," J. Inst. Math. Appl. 25, 241-265 (1980).

27. H. M. Ozaktas and O. Aytür, "Fractional Fourier domains," Signal Process. 46, 119-124 (1995).

28. Z. Zalevsky and D. Mendlovic, "Fractional Wiener filter," Appl. Opt. 35, 3930-3936 (1996).

29. Z. Zalevsky, D. Mendlovic, and J. H. Caulfield, "Localized, partially space-invariant filtering," Appl. Opt. 36, 10861092 (1997).

30. Y. Zhang, B.-Z. Dong, B.-Y. Gu, and G.-Z. Yang, "Beam shaping in the fractional Fourier transform domain," J. Opt. Soc. Am. A 15, 1114-1120 (1998). 